\title{
El regionalismo centroamericano: entre la unión y la integración
}

Gabriel Aguilera Peralta*

RESUMEN

El trabajo examina la experiencia de la región centroamericana, constituida inicialmente por Guatemala, El Salvador, Honduras, Nicaragua y Costa Rica, como una entidad integrada bajo la colonia española, una federación durante sus primeros años de vida independiente, su separación, la constitución de las repúblicas actuales y los repetidos esfuerzos realizados en un comienzo para reconstituir una unión $y$, posteriormente, para una integración. Se examina en particular el rol del Sistema de Integración Centroamericano (SICA), analizando las etapas por las que ha atravesado, los diversos paradigmas que han guiado el proyecto, su proyección en la arena multilateral, sus problemas y perspectivas actuales. Se sostiene que su modelo de regionalización es específico ya que, a diferencia de otros modelos de integración, el centroamericano se distingue por tener como raíz una experiencia histórica de unidad. La discusión se ubica en el marco del proceso de globalización.

Palabras clave: Centroamérica, integración, sICA, regionalismo.

\section{Central American regionalism: between union and integration}

\begin{abstract}
This article examines the experience of the Central American region, constituted at first by the countries of Guatemala, El Salvador, Honduras, Nicaragua and Costa Rica; from its beginnings as an entity integrated under the Spanish colony, a federation during its first years of independent life, their separation and the constitution of the present republics, and the repeated efforts to reconstitute a union
\end{abstract}

* Politólogo. Embajador de Guatemala ante la Organización de Estados Americanos (oEA). aguileraperaltag@gmail.com

Recibido: 9 de octubre de 2015/ Modificado: 13 de mayo de 2016/ Aceptado: 7 de junio de 2016

Para citar este artículo

Aguilera Peralta, G. (2016). El regionalismo centroamericano: entre la unión y la integración. OASIs, 24, 89-105.

DOI: http://dx.doi.org/10.18601/16577558.n24.06 
initially and to achieve an integration later. The experience of the Central American Integration System (SICA) is examined, analyzing the stages of its development, the diverse paradigms that have guided the project, their projection in the multilateral arena, and their present problems and perspectives. It is affirmed that unlike other models of integration, the Central American model is distinctive because it is rooted in a historical experience of unity. The discussion of the process is located within the framework of globalization.

Keywords: Central America, integration, SICA, regionalism.

\section{REGIONALISMO Y GLOBALIZACIÓN}

El regionalismo es un concepto dinámico que reviste multitud de definiciones y de entendimientos conceptuales. Aunque región es inicialmente un concepto geográfico, su uso en las ciencias sociales refiere a la dinámica de Estados y pueblos con diversos niveles de vinculación. En su clásica definición, Nye entiende a la región como un "número limitado de Estados unidos por una relación geográfica y un grado de interdependencia” (Nye, 1968, p. XII) Según Serbin, las regiones podrían ser entendidas como "formas intermedias de comunidad entre las comunidades de los Estados nacionales y la comunidad potencial de la humanidad" (Serbin, s. f. p. 15).

El regionalismo sería, a su vez, el proceso por el cual los Estados y pueblos de una región van integrándose económica, social y políticamente, llegando en algunos casos a conformar una entidad protoestatal diferen- ciada de las individualidades originales y que pueden generar una ideología propia.

No se pueden entender los procesos actuales de regionalismo sin ubicarlos en el marco de la globalización, proceso entendido principalmente como globalización de los mercados, lo que tiene como efecto el predominio de la economía sobre la política y del mercado sobre el Estado, proceso caracterizado también por la revolución de las comunicaciones, la tendencia a la homogeneización de la cultura según los patrones occidentales y de su paradigma político democrático-liberal. Así, la globalización tiende a sustituir las sociedades nacionales por una sociedad internacional interdependiente y multivinculada.

Por ello, el regionalismo puede ser entendido como un elemento de globalización o como una respuesta o alternativa a la misma, esto último en cuanto una reacción a los que se perciben como efectos negativos de la globalización en cuanto acrecentamiento de las desigualdades, la exclusión y la pobreza (Serbin, s. f., p. 16). En el primer supuesto, el regionalismo puede ser un proceso paralelo o superpuesto a la globalización, siendo complementario, y en el segundo supuesto, implicar un proyecto alternativo a la globalización neoliberal con un proyecto de régimen internacional más pluralista (Mitelman, 1999, pp. 189-212).

Desde luego, se han dado experiencias de regionalismo previamente a la expansión de la globalización, como seńala Hettne, autor que distingue entre el viejo regionalismo y el nuevo, este último el correspondiente a la globalización, opinando que las principales di- 
ferencias son que el viejo regionalismo corresponde al mundo de la Guerra Fría y el nuevo a un orden multipolar, el viejo regionalismo fue proteccionista en términos económicos y el nuevo es abierto a la economía mundial, y que en tanto el viejo regionalismo se ubicaba en la dinámica de los Estados-nación, el nuevo forma parte de la nueva estructura mundial en la que actúan también actores no estatales (Hettne, 2002, p. 995).

En tanto que el regionalismo, en el nivel de nuestro análisis, expresa una convergencia de intereses, preocupaciones y aspiraciones entre actores Estatales y no estatales, la integración es un proceso concreto que buscar crear las bases jurídicas e institucionales que permiten la actuación conjunta en la arena internacional.

En América Latina se han dado diversas experiencias de regionalismo, las que corresponden al nuevo regionalismo se manifiestan en nueve procesos de integración incluyendo el centroamericano, expresado en el Sistema de Integración Centroamericano (sICA). Ellos pueden ser diferenciados en el sentido que discute Mitelmann.

\section{LA EXPERIENCIA UNITARIA CENTROAMERICANA}

Una característica central del proceso centroamericano es su raíz histórica, ya que cinco Estados de Centroamérica fueron inicialmente una sola unidad administrativa durante la etapa colonial española, conformando la Capitanía General de Centroamérica, dependiente del Virreinato de Nueva Espańa en México, pero con un grado de autonomía. Después de la emancipación de Espańa en 1821, que transcurrió pacíficamente, y como un efecto de la culminación de la guerra de independencia mexicana, tras una fugaz anexión al Imperio Mexicano de Agustín de Iturbide, Centroamérica se declaró nuevamente independiente en 1823, con el nombre de Provincias Unidas de Centroamérica y, un año después, en 1824 adoptaron el nombre de Republica Federal de Centroamérica, formada por los cinco países que habían sido parte de la Capitanía: Guatemala, El Salvador, Honduras, Nicaragua y Costa Rica, a los cuales se unió posteriormente un sexto país, el Estado de los Altos formado por una segregación de Guatemala, la provincia de Quetzaltenango y otras regiones del occidente así como del Soconusco.

En esa época temprana del republicanismo, el modelo por excelencia era el de los Estados Unidos de América, cuya estructura quiso adoptar Centroamérica en alguna forma. De allí la estructura federal con gobiernos en cada uno de los países y uno central ubicado en Guatemala. Ese modelo no ayudó a formar una conciencia nacional superior a la de cada uno de los países. La Federación estuvo en permanente situación de grave crisis económica. La falta de vías de comunicación no permitió el desarrollo de un comercio federal, las diferencias ideológicas propias del siglo XIX entre conservadores y liberales se sumó a las divergencias de intereses de las clases locales dominantes, especialmente entre los comerciantes y terratenientes de Guatemala y sus pares de las provincias. Gran Bretaña, la potencia hegemónica de la era, la impulsó la desarticulación de la Federación, la cual finalmente se disgregó tras prolongadas guerras 
civiles en 1839. Guatemala, el último país en separarse, reabsorbió al Estado de los Altos.

Sin embargo, a lo largo del siglo xIx y primera mitad del siglo $\mathrm{xx}$, se sucedieron diversos intentos de restablecer la Unión, algunos por medios militares como lo intentó el presidente guatemalteco Justo Rufino Barrios en 1879, y otros mediante acuerdos políticos estatales como la República Mayor de Centroamérica impulsada por el presidente nicaragüense José Santos Zelaya en 1898 y la Republica Federal de Centroamérica creada efímeramente en $1921^{1}$.

Todos esos intentos, como señala el historiador Rafael Cuevas Molina, no fueron esfuerzos de integración sino de restablecimiento de la Unión que había existido (Cuevas Molinas, 2014, p. 26), pero fracasaron porque los problemas originarios que hicieron no viable la experiencia aún no se habían superado por el paso del tiempo, sino más bien se habían intensificado, en especial se había consolidado la identidad nacional de cada uno de los cinco países.

Sin embargo, ese pasado común hizo que persistiera un espíritu de regionalización entre los cinco países que habían formado la Unión.

\section{EN BUSCA DE LA INTEGRACIÓN.}

\section{LA ODECA Y EL MERCOMUN}

Como se sabe, al finalizar la Segunda Guerra Mundial se creó como organismo de las $\mathrm{Na}$ ciones Unidas la Comisión Económica para América Latina (CEPAL), entidad que propuso a los países latinoamericanos una estrategia de desarrollo basada en la industrialización mediante la sustitución de importaciones y un rol conductor del Estado en las políticas económicas, incluyendo la reforma agraria. Esas tesis originales cepalinas eran de inspiración keynesiana. El paradigma incluía la idea de vinculación de pequeñas economías para lograr escalas que permitieran la sustitución de importaciones mediante la ampliación de mercados internos que sustentaran industrias sustitutivas.

Los países centroamericanos asumieron dentro de esas concepciones nuevamente el imaginario de la unidad, pero esta vez expresado como un proyecto de integración regional, por consiguiente, un modelo en el marco del regionalismo. Tuvo influencia en el proyecto la experiencia de la incipiente Comunidad Europea, el principal proyecto de integración de la época.

Por otra parte, y a diferencia de los intentos del pasado, se tuvo presente la importancia de la dimensión económica, en atención a que la mayoría de interpretaciones sobre los fracasos de los intentos de Unión del pasado ubican como una de las causas principales la falta de una base económica común que uniera a los países de la región.

Ambas ideas dieron origen al proyecto integracionista que se inició a mediados del siglo xx. La dimensión política se concretó en la fundación de la Organización de Estados Centroamericanos (odecA) mediante la Carta de San Salvador de 1951, a propuesta del

Análisis del periodo en Herrarte González (1963). 
Gobierno de El Salvador y la económica en el Mercado Común Centroamericano que se inició con el Protocolo de Managua de 1960, mediante el Tratado General de Integración Económica Centroamericana.

La ODECA, cuya carta fue reformada en 1962 en una reunión en Panamá, definió como su objetivo la creación de una comunidad económico-política que aspiraba a la integración de Centroamérica, estableciendo una serie de estructuras regionales que deberían ir dando forma a la institucionalidad supranacional, no solamente con referencia al organismo ejecutivo, sino también al legislativo y al judicial, abarcando los ámbitos educativos, culturales, socioeconómicos y de la defensa. Se creó así una estructura que comprendía: Reunión de jefes de Estado, Consejo de Ministros de Relaciones Exteriores, Consejo Ejecutivo (Secretaría General) Consejo Legislativo (representaciones de los órganos legislativos de los países), Corte de Justicia Centroamericana, Consejo Económico (con los ministros de Economía), Consejo Cultural y Educativo y Consejo de Defensa Centroamericano. (condeca). Se fijó como sede de la Secretaría la ciudad de San Salvador en El Salvador.

La dimensión económica del nuevo proyecto se inició durante en el Iv Periodo de Sesiones de la (CEPAL) en 1951, durante el cual se acordó, a solicitud de los gobiernos centroamericanos, la formación de un Comité de Cooperación Económica del Istmo Centroamericano -el cual se reunió en Tegu- cigalpa, Honduras, del 23 al 28 de agosto de 1952-, iniciándose así el proceso de integración económica con el objetivo de formar un mercado común centroamericano buscando integrar las economías de Centroamérica en forma gradual y progresiva. El esfuerzo culminó con la suscripción del ya mencionado Tratado General de Integración Económica Centroamericana, suscrito el 13 de diciembre de 1960, en Managua, Nicaragua. Ese instrumento creó un Consejo Económico Centroamericano integrado por los ministros de Economía de cada uno de los Estados miembros; la Secretaría Permanente de Integración Económica Centroamericana (siecA), con sede en Guatemala, y el Banco Centroamericano de Integración Económica (BCIE), con sede en Tegucigalpa, Honduras. Se fijó como meta llegar a un Mercado Común Centroamericano en un plazo de cinco años. Otra disposición importante del Tratado fue la de establecer en un plazo breve la unión aduanera $^{2}$. La dimensión económica de la integración se identifica como Mercado Común Centroamericano (MCCA), aunque el mismo no ha llegado a conformarse.

La odeca establecida según la Carta de 1951 no alcanzó a consolidarse debido a conflictos políticos. El Gobierno de Guatemala, bajo el presidente Jacobo Arbenz Guzmán, tenía una orientación de izquierda, impulsando cambios estructurales, incluida la reforma agraria, y su política exterior era no alineada, incluso confrontativa con Estados Unidos. Ello difería centralmente de las posiciones

2 Sobre ese periodo véase Pellandra y Fuentes (2011). 
de los restantes países centroamericanos, que compartían la orientación anticomunista propia de la Guerra Fría.

El conflicto se precipitó cuando Estados Unidos organizó una invasión para derrocar el Gobierno de Arbenz con el apoyo de Honduras y Nicaragua en $1954^{3}$.

El presidente Arbenz fue derrocado y ello restableció el desarrollo de la ODECA, dado que los gobiernos de sus Estados miembros eran mayoritariamente de derecha. En los años subsiguientes, la institución impulsó facilidades de tránsito de personas y de intercambios culturales, así como políticas que buscaban reavivar el sentimiento centroamericanista.

Por su parte, la dimensión económica representada por MCCA fue decisiva para el avance de la integración, dando razón a la consideración de que los anteriores intentos unionistas habían fracasado, además de las razones políticas, porque no existía una base económica común. Aunque no se llegó a la meta -en todo caso irreal- de constituir realmente el mercado común en un lustro, sí se avanzó en otros sentidos: constituir una zona centroamericana de libre comercio y un arancel centroamericano uniforme, todo ello con base en las tesis cepalinas de promover la industrialización para no depender de la exportación de materias primas, y de la creación de espacios protegidos para lograr la sustitución de importaciones.

El resultado fue un avance sustancial del comercio intercentroamericano y el for- talecimiento de la capacidad productiva, así como el inicio de la industrialización de las economías regionales. Ello coincidió con un periodo de alza de demanda para las commodities y varios años de crecimiento económico. Así, el comercio intrarregional se elevó de 30,30 millones de dólares en 1960, a 1,129 en 1980 (sieca, 2012, p. 8). Durante los primeros 10 ańos del MCCA se experimentó un significativo crecimiento económico, con una tasa de crecimiento del producto interno bruto (PIB) y del comercio intrarregional del $5 \%$ promedio anual.

Pero nuevamente factores políticos afectaron el proceso. Diferencias sobre cuestiones migratorias y disputas fronterizas entre El Salvador y Honduras escalaron hasta llegar a una situación de guerra entre ambos países en 1969. Ese conflicto, conocido como la guerra de los 100 Días o la guerra del Fútbol paralizó el desarrollo de la oDECA y afectó, aunque no destruyó, el MCCA ${ }^{4}$.

En 1976, los países buscaron salir de esa situación dando un salto adelante. El Comité de Alto Nivel (CAN), compuesto por representantes de los países miembros de ODECA, elaboró el proyecto de Tratado de la Comunidad Económica y Social Centroamericana que preveía llegar a la plena integración en un plazo de 25 años, estableciendo como metas intermedias la unión aduanera, la unión monetaria, la armonización tributaria, la libre circulación de trabajo y servicios y una política económica común.

Sobre esos acontecimientos véase Cullather (1999).

4 Sobre la guerra de los 100 Días, véase Andersson (1981). 
Sin embargo, ese proyecto no avanzó por la situación política en el Istmo. Para la década de los setenta, la mayoría de los gobiernos de la región eran autoritarios de derecha y militarizados. La ausencia de derechos democráticos y violaciones de los derechos humanos, así como la vigencia de las ideologías de mediados del siglo $\mathrm{xx}$, originaron rebeliones armadas que fueron vinculadas al contencioso entre los grandes actores de la Guerra Fría. El triunfo de la Revolución Sandinista y la subsiguiente guerra "Contra" en Nicaragua, las guerras revolucionarias en Guatemala y El Salvador, y el involucramiento de Honduras y El Salvador en esos conflictos, así como la intervención de Estados Unidos por una parte y de Estados del Pacto de Varsovia por otra, crearon una situación caótica y de extrema violencia que paralizó los esfuerzos integracionistas entre los años setenta y ochenta del siglo $\mathrm{xx}^{5}$.

El balance de ese cuarto de siglo de integración indica que fue correcto y acorde a la época el haber abandonado el imaginario del siglo XIX y primera parte del xx de que se podía restablecer la Unidad de los cinco países por decisión política de los Estados, sin atender a la realidad económica y al hecho central de que se habían consolidado identidades nacionalitarias más fuertes y vinculadas a la realidad, que el vago ideal de una unidad que había existido décadas antes y, por consiguiente, haber girado a la construcción de un proyecto integracionista que planteaba la construcción pausada y realista de una identidad regional con un componente central en el segmento económico representado por el Mercado Común Centroamericano en el marco del paradigma cepalino de la época y del viejo regionalismo.

La experiencia fue positiva en la medida que se logró crear un Proyecto y una institucionalidad regional, y que el componente económico alcanzó su objetivo de vincular a las economías de Centroamérica y, por consiguiente, hacerse atractivo para las élites sociales y económicas. Empero, las ambiciosas metas originales en el marco económico no se cumplieron. Pese a su nombre, el Mercado Común Centroamericano no fue tal, ya que si bien logró inicialmente alcanzar con cierta rapidez la creación de una zona de libre comercio, no pudo conformar la unión aduanera y mucho menos el mercado común propiamente dicho.

Sin embargo, los conflictos políticos y militares impidieron el avance del proyecto y finalmente paralizaron del todo a la ODECA en cuanto segmento político. En ese sentido existe una interesante semejanza con las causas del fracaso de la Unión en el siglo XIX. No obstante, los mecanismos del mCca en lo esencial se mantuvieron.

\section{LA ODECA SE CONVIERTE EN EL SICA}

Entre finales de los años ochenta y principios de los noventa del siglo pasado concluyo la Guerra Fría con la desintegración de la Unión Soviética y la caída del muro de Berlín. Ello creó un escenario favorable al progreso de iniciativas que buscaban impulsar la pacificación

Para un análisis del periodo véase Torres-Rivas (2011). 
y la democratización de Centroamérica. Los esfuerzos del Grupo de Contadora primero, y después los de Esquipulas I y II, iniciativa del entonces presidente de Guatemala Vinicio Cerezo, permitieron acuerdos que pusieron fin a los conflictos intercentroamericanos. A la vez, procesos de negociación permitieron que se llegara a la paz en El Salvador, Nicaragua y por último en Guatemala, finalizando las largas guerras internas. En ese mismo ambiente se dieron transiciones a la democracia y la conclusión de los gobiernos autoritarios militares ${ }^{6}$. Se dieron así las condiciones para un relanzamiento del proceso de integración.

El primer paso se dio durante la reunión de Esquipulas I en 1986, cuando los presidentes centroamericanos acordaron la creación de un Parlamento Centroamericano (parLacen). Con ello se constituyó un Foro que propició el diálogo integracionista y que creó las condiciones para que posteriormente, durante la XI cumbre presidencial del año 1991, se acordara el Protocolo de Tegucigalpa que reformó la Carta de la odeca dando origen al Sistema de Integración Centroamericano (sICA), una nueva estructura institucional conformada por los cinco países originales más Panamá.

El sica se diseñó con los siguientes órganos:

- La Reunión de Presidentes

- La Reunión de Vicepresidentes

- El Parlamento Centroamericano

- La Corte Centroamericana de Justicia

6 Sobre ese proceso véase Arson (1999).
- El Consejo de Ministros de Relaciones Exteriores

- El Comité Ejecutivo del sica

- La Secretaría General del sica

- El Comité Consultivo del sica

Esa estructura amplía y profundiza el diseńo de órganos que tenía la antigua ODECA, aunque no todos los países se han incorporado a la Corte Centroamericana o al parLaCEN, lo cual expresa una política que permite a los países miembros no adherirse a los instrumentos que no coincidan con sus políticas nacionales, esto hace que el proceso integracionista tenga en su seno diversas velocidades, con unos países avanzando más que otros.

Una novedad en la estructura reformada fue la creación del Comité Consultivo, ente en el que participan representantes de la sociedad civil, lo que busca incentivar el interés de la sociedad centroamericana en el proceso, al darles la oportunidad de participar e interactuar con los gobiernos.

La declaración de principios del sica, establecida en el Protocolo de Tegucigalpa, incluye la realización de la integración de Centroamérica para constituirla como Región de Paz, Libertad, Democracia y Desarrollo. Se propone el fortalecimiento de la democracia, la seguridad regional, el bienestar y la justicia económica y social, la unión económica y la conformación de la región como bloque económico, la promoción del desarrollo económico, social, cultural y político, y la defensa del medio ambiente (SICA, 1998a). 
La denominación de "Sistema" que se dio a la organización refleja la concepción teórica sistémica que inspiró la nueva arquitectura. En la misma, la organización se estructuraba sobre cinco subsistemas, cada uno de ellos con un tratado u otra norma propia. Los subsistemas fueron originalmente: Político, Económico, Social, Ambiental y Cultural y Educativo. Se entiende que el Protocolo de Tegucigalpa, al crear el sICA, estableció el subsistema Político.

El subsistema de Educación y Cultura fue uno de los primeros, establecido mediante el Convenio Constitutivo de la Coordinación Educativa y Cultural Centroamericana en 1982. Le siguió el subsistema Ambiental, definido en el Convenio Constitutivo de la Comisión Centroamericana de Ambiente y Desarrollo (CCAD) de 1989.

Por otra parte, se buscó definir un marco ideológico del proyecto que se impulsaba. El mismo se definió en 1994, cuando una cumbre presidencial en Managua, Nicaragua, adoptó la declaración de la Alianza para el Desarrollo Sostenible (ALIDES) como base para el modelo de desarrollo regional. En ese modelo se integran la democracia, el desarrollo sociocultural y económico, el manejo sostenible de los recursos naturales, la mejora de la calidad ambiental y el equilibrio ecológico.

En otra cumbre un año después, en 1995, los mandatarios firmaron en San Salvador el Tratado de Integración Social Centroamericano, que procura la integración social de Centroamérica y que establece otro subsistema, el Social.

Ese mismo año, en la Cumbre Presidencial de San Pedro Sula, Honduras, se trató el tema de la seguridad regional suscribiéndose el Tratado Marco de Seguridad Democrática en Centroamérica, el cual, a su vez, instituye la Comisión de Seguridad de Centroamérica. El Tratado introduce el concepto de seguridad democrática, entonces en boga, en contraposición al de seguridad nacional que primó durante la Guerra Fría. La seguridad democrática vincula la seguridad con la democracia, el respeto por los derechos humanos, el fortalecimiento del poder civil e introdujo el concepto de la seguridad multidimensional que posteriormente adoptó la oEA en la Conferencia Especial de Seguridad en México, en el año 2003. Ello significo la creación del subsistema de Seguridad.

En el campo de la dimensión económica, en 1993 se firmó el Protocolo de Guatemala al Tratado General de Integración Económica Centroamericana, que organiza el subsistema de Integración Económica de acuerdo con la nueva estructura del sica. En ese Tratado se establece que su objetivo es el desarrollo económico y social equitativo y sostenible de los países centroamericanos, el cual debe generar el bienestar de sus pueblos y el crecimiento de los países miembros, por medio de un proceso que permita la transformación y modernización de sus estructuras productivas, sociales y tecnológicas, que eleve la competitividad y logre una reinserción eficiente y dinámica de Centroamérica en la economía internacional. Su ente ejecutor es la Secretaría de Integración Centroamericana (SIECA) con sede en Guatemala. Este es el subsistema económico (sICA, 1998b).

La conformación del sica dio un impulso renovado a la integración. La importancia de Centroamérica en el campo económico y 
político aumentó e incluso generó su expansión geográfica. Panamá, que no formó parte original de las uniones del siglo XIx debido a que pertenecía a Colombia, se unió primero al segmento político del SICA y posteriormente a su segmento económico. Belice, país originado en la experiencia colonial británica y con el cual Guatemala mantiene una antigua controversia territorial y marítima, también se integró al segmento político y finalmente hizo lo mismo la República Dominicana, a pesar de que este país caribeño no tiene pasado común ni continuidad geográfica con el istmo centroamericano. En este último caso primó el análisis de las ventajas de pasar a formar parte de una integración regional con países de economías de escala similar y adherirse a las ventajas del actor amplificado. Actualmente, la República Dominicana examina la posibilidad de incorporarse al Subsistema económico.

Así ampliada, la población del sica pasó a constituir el $9 \%$ de la población de Latinoamérica, con 53'598.514 millones de habitantes en el 2013 con un piв de us $\$ 417$ billones.

Empero, debe distinguirse la existencia de dos subbloques en el seno del sica ampliado. Por una parte, el grupo constituido por el CA 4 -los países que son miembros de todos los órganos y tratados de la integración y que han avanzado en la libre circulación de personas, formado por Guatemala, El Salvador, Honduras y Nicaragua-, y el grupo originalmente del CA 3 -Costa Rica, Panamá y Belice-, Estados que escogen a cuáles de los órganos y tratados se incorporan. A este segundo grupo se ha incorporado República Dominicana.

Esta diferencia obedece a distintas razones. En el caso de Costa Rica, aunque partici- pó en la unión original y mantuvo adhesión a los diversos intentos históricos de restablecerla, su régimen político específico, de tradición democrática liberal, no existencia de ejército e indicadores socioeconómicos más altos que el resto de Centroamérica, hace que sus intereses nacionales sean predominantes en la relación con el sica y que se preste atención a que la integración con Centroamérica no afecte el modelo costarricense, lo que explica su tardía incorporación a la moderna integración. Por ello el país no se ha incorporado al parlacen ni a la Corte Centroamericana de Justicia. Los otros tres Estados: Belice, Panamá y República Dominicana han tenido una formación histórica y un desarrollo cultural distinto al resto de Centroamérica, y por ello su incorporación al proyecto centroamericano no parte de antecedentes históricos, sino de consideraciones sobre las ventajas de incorporarse a un decurso de regionalización que potencia su participación en la arena internacional.

Por otra parte, en el sICA existe la figura de países observadores regionales y extrarregionales; actualmente se han incorporado 24 Estados de diversas regiones del mundo incluyendo Latinoamérica, Europa y Asia.

El sICA mantiene una relación particular con México y Colombia, países con los cuales comparte un proyecto de desarrollo regional común, el Proyecto Mesoamérica, así como diversos arreglos de seguridad.

A la par de los procesos de integración formales se ha desarrollado un proceso informal en la medida que los conglomerados empresariales han extendido sus operaciones a países de la región y que los actores de la 
sociedad civil también han aumentado sus redes de relación.

\section{EL SICA Y LA GLOBALIZACIÓN}

Los primeros lustros posteriores al fin de la Guerra Fría parecieron consagrar la hegemonía mundial de Estados Unidos de América y su concepción de que la democracia liberal de mercado se convertiría en un modelo mundial. Consecuentemente, la influencia norteamericana se extendió por América Latina buscando orientar los órdenes económicos de acuerdo con los dictados de lo que se conoció como el Consenso de Washington, conjunto de diez recomendaciones que se estimaban válidas para los países en desarrollo. Esas recomendaciones o fórmulas, elaboradas por el Fondo Monetario Internacional, el Banco Mundial y el Departamento del Tesoro de Estados Unidos, contenían políticas económicas relativas a la estabilización de la macroeconomía, la liberación de las económicas nacionales y del comercio, el fomento de las inversiones, recomendaban las medidas de ajuste estructural $y$, en general, promulgaban la idea de que las fuerzas del mercado impulsarían el desarrollo. A nivel teórico, Francis Fukuyama buscó darle sustento ideológico con su famosa fórmula del fin de la historia.

En ese entorno, el paradigma cepalino originario de la integración centroamericana se hizo inaplicable; lejos de buscar la creación de espacios económicos protegidos para fomentar la industrialización propia y los mercados internos con intervención del Estado, el Consenso planteaba todo los contrario: abrir los espacios económicos nacionales a las fuerzas del mercado internacional con el libre flujo de bienes, servicios y capitales, reducir al máximo al papel del Estado en la economía, privatizar los medios de producción estatales, mantener al máximo la estabilidad macroeconómica sin consideración de sus efectos sociales, y estimar que las inversiones y la creación de empleos impulsarían el desarrollo. Esto significó el paso al nuevo regionalismo.

El sICA buscó adaptarse al nuevo orden económico internacional, que ya era el de la globalización y, a la vez, preservar su propia integración. Lo hizo adoptando el nuevo paradigma de la CEPAL conocido como "regionalismo abierto", fórmula híbrida que buscaba conservar la interdependencia derivada de los acuerdos comerciales preferenciales contenidos en los acuerdos de integración con la interdependencia nacida de la dinámica de los mercados internacionales, aceptando que para lograr el desarrollo se requería elevar la competitividad. Al contrario de las formulaciones pasadas, esta vez se requería la rebaja de aranceles y la apertura de los mercados nacionales al comercio internacional (CEPAL, 1994).

El nuevo modelo tiene como uno de sus ejes centrales los acuerdos de libre comercio. En su oportunidad, Estados Unidos impulsó el proyecto del área de Libre Comercio de las Américas (ALCA) que pretendía convertir a la totalidad del continente americano en un espacio de comercio libre y preferencias arancelarias. Ese proyecto no se realizó pero fue sustituido por múltiples tratados bilaterales o multilaterales.

Los más importantes han sido el DRCAFTA que estableció el libre comercio entre Centroamérica, República Dominicana (que 
para la época de suscripción no se había integrado al SICA) y los Estados Unidos de América, así como los TL Centroamérica-México, Centroamérica-República Dominicana, CAPanamá, CA-Chile. Aparte de ello, los países del sICA han suscrito bilateralmente varios TLC y Acuerdos de Alcance Parcial.

Esa modalidad afectó la esperada secuencia de desarrollo de la integración económica CA hacia un Mercado Común. En especial, la Unión Aduanera Centroamericana y la consolidación de un arancel común dado que los diversos TLC suscritos por los países del SICA establecieron diferentes plazos y condiciones para las desgravaciones, lo que provocaría que la Unión Aduanera debería esperar la conclusión de los esos plazos para ser posible (Caldentey, 2014, p. 156).

Una excepción entre el conjunto de TLC es el Acuerdo de Asociación CA-Unión Europea, ya que ese instrumento, que en realidad consta aparte de un TLC de dos pilares, uno de cooperación y otro de diálogo político, contiene un capítulo específico con compromisos en integración regional que promueven el avance de la integración, entre ellas la obligación de establecer una Unión Aduanera en un plazo de cinco años y la incorporación de Panamá al segmento económico de la integración CA. A raíz de ello se dieron algunos avances en la Unión Aduanera entre Guatemala y Honduras.

En el espacio de tiempo en que el sICA optó por el regionalismo abierto, se estaban desarrollando otras modalidades de regiona- lismo latinoamericano. Algunas constituyeron modalidades de integración para avanzar hacia uniones aduaneras y políticas económicas comunes como Can, CARICOM y MerCosur. Pero otras plantearon un paradigma de integración alternativo. Esta modalidad, conocida como regionalismo posliberal, retoma el papel del Estado en el desarrollo, la construcción de espacios propios, el énfasis en los temas sociales del combate a la pobreza y la desigualdad, y se extiende a generar espacios de autonomías regionales en la arena internacional, en algunas de sus manifestaciones confrontando la hegemonía norteamericana.

Esos acuerdos dieron origen al CELAC, unasur, alba-Tratado de Comercio de los Pueblos ${ }^{7}$. Ello fue políticamente posible por el ascenso de fuerzas de izquierda que llegaron al poder en varios de esos Estados. Esa modalidad de regionalismo alternativo, sin embargo, se ha debilitado a partir de cambios políticos en varios de los países que lo impulsaban, en lo que parece ser un retorno al predominio de gobiernos favorables al paradigma neoliberal.

Los Estados del sica no optaron por el modelo alternativo, a pesar de que dos gobiernos de la región son de izquierda y uno de ellos pertenece a ALBA. La razón es el alto nivel de vinculación que el sica y los Estados que la componen tienen con Estados Unidos; este país es el primer socio comercial de la región, uno de los principales inversores y proveedores de cooperación, de turismo y, fundamentalmente, el receptor de la gran mayoría de emigrantes centroamericanos.

Sobre el regionalismo posliberal véase Serbin (2012). 
Cuenta igualmente la diferencia en problemática social del sica en relación con las integraciones del Sur. La violencia criminal es una de las principales amenazas a la gobernabilidad y al desarrollo democrático, y aunque afecta a toda la región, es especialmente grave en Guatemala, El Salvador y Honduras (conjunto de países conocidos como el "Triángulo Norte"). El aumento del crimen organizado se debe especialmente a la acción de los carteles dedicados al narcotráfico, que promueven actividades criminales colaterales como la trata de personas, el robo de automóviles, el lavado de activos y otros delitos, constituyendo verdaderas corporaciones criminales, con capacidad de infiltración en los organismos del Estado. Se estima que el $88 \%$ de la cocaína que llega al mercado norteamericano transita por Centroamérica, lo que indica la importancia de la región para la narcoactividad (UNDCCP, 2010, en Benítez, 2010, p. 15).

La otra causa de la violencia es el fenómeno de las pandillas juveniles conocidas como "Maras", que alcanzan niveles muy altos en el "Triángulo Norte". Las Maras son mutaciones en Centroamérica del pandillerismo norteamericano, introducido en los ańos noventa por el movimiento migratorio de jóvenes que por razones sociales tales como la desintegración familiar, la ausencia de suficientes oportunidades de acceso a la educación y a ocupación del tiempo libre, el desempleo juvenil y la pobreza aumentaron desmesuradamente, vinculándose en algunos casos a los carteles de la droga.

Las Maras, que constituyen una subcultura sustituta de la familia con elaborados códigos de incorporación y pertenencia, ejercen violencia extrema en forma de robos, extorsiones, delitos sexuales y asesinatos, afectando especialmente a los estratos populares y medios de las poblaciones. El elevado número de las pandillas hace difícil a los Estados su contención por políticas represivas, a la vez que el origen social de la manifestación genera que solo se puedan tratar eficazmente mediante cambios en políticas sociales de muy largo plazo (Savenje, 2009).

El efecto combinado de las dos formas de violencia es el que la región, nuevamente en especial el "Triángulo Norte”, tengan algunas de las tasas más altas del mundo en muertes por cien mil habitantes (exceptuando las naciones en guerra), con igualmente altos indicadores de sensación de vulnerabilidad y de deterioro de la calidad de vida. Los costos de la violencia impactan en el crecimiento económico, reducen el atractivo para inversiones en los países afectados debido al costo agregado de los gastos en seguridad, y generan que un porcentaje importante del PIB se consuma en este aspecto.

Otro de los efectos de la violencia, aunado a la pobreza, el desempleo y la búsqueda de reunificación familiar es el fenómeno de la elevada migración de la región principalmente hacia Estados Unidos. Para el ańo 2010, fecha del último censo disponible, se contabilizaron 3'998.280 centroamericanos en Estados Unidos, cifra que se estima ha aumentado considerablemente en el último quinquenio. El flujo de migrantes, principalmente de El Salvador y Guatemala, se ha mantenido y ha aumentado una de sus facetas más graves, el flujo de migrantes menores de edad sin acompañamiento. Para el año 2014 se calcula que 
llegaron a Estados Unidos 60.000 menores solos procedentes de El Salvador, Guatemala y Honduras ${ }^{8}$.

Dado que la mayoría de migrantes se trasladan sin visa empleando rutas terrestres, corren elevados riesgos a su integridad y vida por lo azaroso de las rutas, particularmente del desierto, y por los ataques de bandas delincuenciales que les roban y extorsionan, en tanto que las mujeres son frecuentemente víctimas de abusos sexuales y de ser sometidas a esclavitud. Los que logran llegar a la Unión americana trabajan en condiciones precarias por la falta de documentos y son deportados en gran número. De allí que un tema central en la agenda de relaciones de países centroamericanos con Estados Unidos es la gestión para la seguridad y legalización de los migrantes.

Desde la perspectiva norteamericana, el problema de la inmigración indocumentada es un asunto de seguridad. Sus autoridades consideran a Centroamérica una de las cinco prioridades en la agenda de política exterior, a la par de Afganistán, Irak, Siria y Ucrania.

Desde 2015 se ha venido trabajando en un programa destinado a estimular el desarrollo económico y humano en las zonas de los países del "Triángulo Norte", de donde más emigran los habitantes. El llamado el Plan de Alianza para la Prosperidad es promovido conjuntamente por los países del "Triángulo Norte” y Estados Unidos; está previsto para una duración de 5 ańos y un presupuesto que puede llegar a los 7.900 millones de dólares, de los cuales Estados Unidos podría aportar inicialmente cerca de mil millones (Prensa Libre, 2015).

\section{CONCLUSIÓN: SALTAR AL FUTURO}

Pese a sus logros, el proyecto integracionista centroamericano, a 65 años de su inicio, acusa un ritmo de desarrollo lento y en alguna forma repetitivo. Su subsistema Económico sigue intentando armar la Unión Aduanera, una meta que ya se intentaba alcanzar desde la época de la odecA. El Parlamento Centroamericano, en alguna forma similar al Parlamento Europeo por la elección directa de sus diputados, no impacta mayormente en el proceso porque sus decisiones no son vinculantes. Tiene utilidad como un foro de discusión y propuestas políticas regionales, pero carece de capacidad legislativa. La Corte Centroamericana de Justicia tiene jurisdicción solamente sobre cuatro de los países miembros del sica. En el segmento político se critica que pese al nutrido programa de cumbres y de reuniones de todo tipo, así como del enorme número de resoluciones y de una institucionalidad extendida y que continúa reproduciéndose, no se observa avance en la formación de una construcción estatal supranacional o de una identidad centroamericana que vaya sobreponiéndose a las nacionales, teniendo en cuenta que el imaginario del proyecto no es una mera integración sino llegar a la Unión.

8 Según declaraciones del consejero del Departamento de Estado de Estados Unidos Thomas Shannon. Vease Agencia efe (2015). 
Los problemas son, por una parte, de falta de voluntad política y, por la otra, técnicoadministrativos. En diversas ocasiones se han iniciado en el sica esfuerzos de reforma para acelerar el proceso. El actual fue acordado en la Cumbre de San Salvador de julio del 2010, con el llamado "relanzamiento del proceso de integración centroamericana” que contiene cinco ejes de acción: seguridad democrática, prevención y mitigación de los desastres naturales y de los efectos del cambio climático, integración económica y fortalecimiento de la institucionalidad regional. Esa declaración se acompañó de un Plan de Acción y de elevar esfuerzos para implementar en su totalidad la Estrategia de Seguridad de Centroamérica y México, la Estrategia de Cambio Climático, la Agenda Estratégica Social y nuevamente la Unión Aduanera'. Sin embargo, en su primer quinquenio de aplicación los avances han sido lentos.

Otro factor disfuncional es la persistencia de conflictos fronterizos, territoriales y marítimos entre los países de la región. Con excepción de Panamá y del nuevo integrante, República Dominicana, todos los demás Estados del SICA tienen o han tenido diversos tipos de contenciosos entre sí. Algunos se han resuelto mediante fallos de la Corte Internacional de Justicia pero otros, como los diversos conflictos entre Nicaragua y Costa Rica, se mantienen y afectan el diálogo político de alto nivel, y al renovar posiciones nacionalistas ralentizan la formación de un espíritu comunitario.
El balance general después de más de medio siglo de esfuerzos integracionistas es, sin embargo, positivo. A su favor tiene la ODECA el haber llevado la retórica unionista al terreno real. Antes de su existencia, las comunicaciones entre los países de Centroamérica eran precarias, existía poco conocimiento mutuo de la sociedad y escasa comunicación. Esas carencias empezaron a revertir con la ODECA y se empezó a crear el espíritu comunitario. El sicA, por su parte, adaptó el proyecto a la globalización, se amplió con la incorporación de nuevos Estados miembros, lo que evidenció su atractivo y dinamismo, y se consolidó como un acto regional con capacidad de actuar como bloque en la arena internacional. El segmento económico del proceso en sus dos etapas ha vinculado efectivamente a las economías de la región, incluyendo la interconexión eléctrica que se ha consolidado, y si bien la meta de llegar a un mercado común es aún lejana, es realista que se avance en la meta de la Unión Aduanera por ser una exigencia del Acuerdo de Asociación con la Unión Europea. La gestión de las economías centroamericanas vinculadas es igualmente de importancia regional. Cuatro de los Estados gozan de libre movilidad de personas a través del CA-4.

No obstante, queda abierto el interrogante inicial: si el imaginario de restablecer la Unión Centroamericana es aún posible o si, por el contrario, debe considerarse que la realidad del siglo xxi lo que permite es cierto perfeccionamiento del proceso de integración

9 Documentos en SICA (2010). 
en el marco de la globalización y el nuevo regionalismo.

El discurso unionista se mantiene en el seno del sica como un elemento ideológico, pero es debatible si en el marco de la nueva estructura mundial sea posible y realista recrear de los Estados del sica un solo supra-Estado.

\section{REFERENCIAS}

Agencia efe (2015). Consejero del Departamento de Estado de eua cree que Centroamérica es una prioridad. Recuperado de www.efe.com/efe/ america/politica/el-consejero-del-departamentode-estado-eeuu-cree-que-centroamerica-es-unaprioridad/20000035-2670781 - 64k.

Arson, C. (1999). Comparative Peace Process in Latin America. Stanford: Stanford University Press.

Andersson, T. (1981). The War of the Dispossessed, Honduras and El Salvador. Nebraska: University of Nebraska Press.

Benítez Manuat, R. (ed.) (2010). Crimen organizado e iniciativa Mérida en las relaciones México-Estados Unidos. México: CASEDE.

Caldentey, P. (2014). Los desafíos estratégicos de la integración centroaméricana. Estudios y Perspectivas 156.

CEPAL (1994). El regionalismo abierto en América Latina y el Caribe: la integración económica al servicio de la transformación productiva con equidad. Santiago de Chile: Los Libros de la CEPAL.

Cuevas Molina, R. (2014). La Unión Centroamericana en el S. XIx. Rebela, 4 (1).

Cullather, N. (1999). Secret History, The CIAs Classified Account of its Operations in Guatemala, 19521954. Stanford: Stanford University Pres.
Herrarte Gonzales, A. (1963). La Unión Centroamericana. Tragedia y esperanza. Guatemala: Ministerio de Educación Pública.

Hettne, B. (2002). El nuevo regionalismo y el retorno a lo político. Comercio Exterior, 52 (11).

Ianni, O. (1996). Teorías de la globalización. México: Siglo XXI Editores.

Mitelman, J. H. (1999). Rethinking the New Regionalism in the context of Globalization. Global Governance, 2 (2).

Nye, J. (ed.) (1968) International Regionalism. Boston: Little Brown and Company.

Pellanda, A. y Fuentes, J. A. (2011). El estado actual de la integración en Centroamérica. Estudios y Perspectivas 129.

Oman, C. (1999). Globalization, Regionalization and Inequality. En Hurrel, A., Woods, N. (eds). Inequality, Globalization and World Politics. New York: Oxford University Press.

Savenje, W. (2009). Maras y Barras, Pandillas y violencia juvenil en los barrios marginales de Centroamérica. San Salvador: FLACSO.

Serbin, A. (2012). Regionalismo y soberanía nacional en América Latina. Lecciones aprendidas y nuevos desafíos. En Mesa, M. (coord.). El mundo a la deriva: crisis y pugnas de poderes. Madrid: CEIPAZ.

Serbin, A. (s. f.). Globalización, regionalismo y sociedad civil. Buenos Aires: Documentos CRIES.

Sistema de Integración Centroamericana (SICA) (1998a). Protocolo de Tegucigalpa a la Carta de la oDECA. En El Libro de Centroamérica. Un instrumento civico de los pueblos. San Salvador: Secretaría General del sica.

Sistema de Integración Centroamericana (SICA) (1998b). Protocolo de Guatemala al Tratado General de Integración Centroamericana (Protocolo de 
Guatemala). En El Libro de Centroamérica. Un instrumento cívico de los pueblos. San Salvador: Secretaría General del sica.

Sistema de Integración Centroamericana (SICA) (2010). Cumbre extraordinaria de Jefes de Estado y de Gobierno de los países del Sistema de Integración Centroamericana SICA para el relanzamiento del proceso de la integración centroamericana. San Salvador: Centro de Documentación del sica.
SIECA (2012). Estado de situación de la integración centroamericana. Recuperado de: URL.

Stallings, B. (ed.) (1995). Global Challenge, Regional Response, The new International Context of Development. Cambridge: Cambridge University Press. Torres-Rivas, E. (2011). Revoluciones sin cambios revolucionarios. Ensayos sobre la crisis en Centroamérica. Guatemala: Guatemala F y G: Editores. 